\title{
Position-based cavity tuning
}

\author{
N. Milas $\odot,{ }^{1,}{ }^{*}$ C. Plostinar, ${ }^{1}$ R. Miyamoto, ${ }^{1}$ M. Eshraqi $\odot,{ }^{1}$ and Y. Liu $\odot^{2}$ \\ ${ }^{1}$ European Spallation Source (ESS), Lund, Sweden \\ ${ }^{2}$ J-PARC Center, KEK \& JAEA, 2-4 Shirakata, Tokai, Naka, Ibaraki 319-1195, Japan
}

(Received 16 March 2020; accepted 27 October 2020; published 19 November 2020)

\begin{abstract}
Knowledge of the longitudinal beam parameters is important for understanding beam dynamics in any machine. In a linear accelerator (linac), to guarantee a stable motion in the longitudinal plane, the settings for the radio frequency cavities must be established by determining the phase and amplitude witnessed by the beam. In most modern hadron linacs, the requirements on phase and amplitude accuracy are $1^{\circ}$ and $1 \%$ respectively, which is particularly challenging. Although systematic approaches for finding the phase and amplitude of cavities are available in many facilities with operational linacs, it is also vital to have a system in place when the standard time-of-flight based functionality is down or not yet available (as is often the case in the early stages of commissioning). Such a method should be relatively fast and simple, and should not depend on time-of-flight measurements or a rigorous phase calibration of the beam position monitors. This work presents a new phase- and amplitude-scan method which only requires information from transverse positions measured at beam position monitors downstream of the cavity being scanned. The scanning then relies on transverse trajectory correction, which has been developed extensively by the community. As a by-product, this method can also provide estimates for a cavity's transverse misalignment. The method has been developed and tested for a single-cell cavity; the simulated effectiveness for a multicell cavity is also presented.
\end{abstract}

DOI: 10.1103/PhysRevAccelBeams.23.114002

\section{INTRODUCTION}

As new linear hadron accelerators are constructed and commissioned with tighter loss budgets and requirements, the ability to set up the acceleration chain as accurately as possible becomes essential. Setting the correct phase and amplitude for the cavities ensures that the particle bunches receive the desired acceleration and energy gain, thus maintaining the quality of the beam and reducing halo growth and losses. A well-developed, systematic approach for establishing the phase is in use at many facilities [1-7] using time-of-flight (ToF) and measuring the phase differences in beam-position monitors (BPMs) selected downstream of the scanned cavity. Using the same data, it is also possible to calibrate amplitudes with the method known as signature matching [7].

However, comparable tools and diagnostics are not always available, and different approaches are needed. For example, some critical factors include the calibration of cable lengths, the phase relation between different

*natalia.milas@ess.eu

Published by the American Physical Society under the terms of the Creative Commons Attribution 4.0 International license. Further distribution of this work must maintain attribution to the author(s) and the published article's title, journal citation, and DOI.
BPMs, thermal drifts, or other attributes coming from the dynamics of the beam distribution during the tuning.

We present here a unique phase- and amplitude-scan method, using only information from transverse positions measured at a few BPMs selected downstream of the cavity being scanned. In addition to phase and amplitude calibrations, this method can also provide the transverse offsets and tilts (pitch and yaw angles) of the cavities, which can then be fed back to the machine model.

In Sec. II we present the position-based cavity tuning method, including a discussion of how to obtain cavity misalignment from the measured trajectories. In Sec. III, a set of measurements done at the Japan Proton Accelerator Research Complex (J-PARC) is used as a proof-of-principle for the new tuning technique. In Sec. IV, the theory of the position-based phase scan for a single gap versus a multicell cavity is presented, along with a test simulation of the method, error analysis, and discussion. Section V addresses the limitations of the method, with conclusions presented in Sec. VI.

\section{POSITION-BASED CAVITY TUNING}

Consider a simple cavity-drift lattice. When a charged particle traverses a rf cavity it also receives a transverse kick proportional to the initial offset, which may be converging or diverging depending on the phase of the cavity. The amplitude of this effect depends on both the 


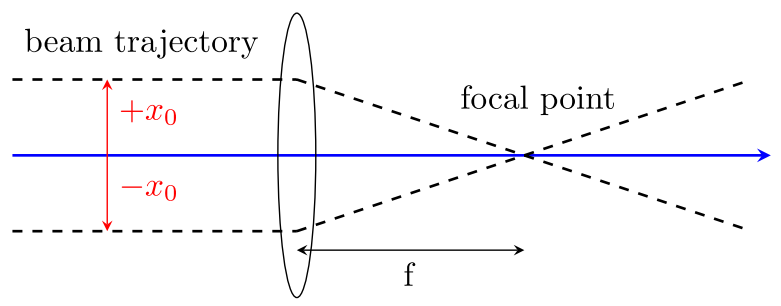

FIG. 1. The trajectory crossing occurs only at the focal point.

phase and amplitude of the cavity, and thus affects the beam trajectory accordingly:

$$
\left[\begin{array}{l}
x \\
x^{\prime}
\end{array}\right]=\left[\begin{array}{cc}
1 & 0 \\
1 / f(\phi, V) & 1
\end{array}\right]\left[\begin{array}{l}
x_{0} \\
x_{0}^{\prime}
\end{array}\right],
$$

where $f(\phi, V)$ is the rf focusing, which for a linear model can be expressed as

$$
\frac{1}{f(\phi, V)}=-\frac{\pi q V T \sin (\phi)}{m c^{2} \lambda(\gamma \beta)^{3}}=F(V) \sin \phi .
$$

Here, $T$ is the cavity transit-time factor, $\lambda$ the rf wavelength, $\phi$ the rf phase seen by the beam and $V=E_{0} L$ is the gap voltage with $L$ is the effective gap length. The mapping of the full lattice is then

$$
\left[\begin{array}{c}
x \\
x^{\prime}
\end{array}\right]=\left[\begin{array}{c}
x_{0}+L_{d} x_{0} F(V) \sin \phi+L_{d} x_{0}^{\prime} \\
x_{0} F(V) \sin \phi+x_{0}^{\prime}
\end{array}\right]
$$

where $L_{d}$ is the length of the drift between the cavity and the observation point, which in most cases will be a BPM and will be referenced as such from this point on. We show that by scanning the cavity phase and examining the trajectory it is possible to calibrate the phase and amplitude of the rf fields.

One way to calibrate the cavity amplitude is to run several phase scans for different amplitudes and record the maximum trajectory displacement; this occurs when the cavity phase is at maximum focusing or defocusing in the transverse plane, that is, at 90 and -90 degrees from the linac definition of zero phase. The trajectory deviation is a function of the cavity amplitude-from the second term for the trajectory in Eq. (3), $\Delta x_{\max }=L_{d} x_{0} F(V)$. The variables $L_{d}$ and $x_{0}$ are known parameters and the function $F(V)$ can be calculated from the model using the cavity field maps. A relation between the amplitude of the $\mathrm{rf}$ field witnessed by the beam and the power input from the klystron can then be calculated.

Another way to calibrate the amplitude, which is easier to visualize despite requiring a more difficult setup, is to measure the real transverse focal length of the cavity as shown in Fig. 1. In this method the initial trajectory angle has to be correctly set to zero and it is also assumed that a previous beam-based alignment for the nearby BPMs was performed and the remaining trajectory errors are small [8].
An amplitude scan is then measured for two initial trajectory offsets $x_{10}=-x_{20}=x_{0}$ and $x_{10}^{\prime}=x_{20}^{\prime}=0$. The crossing point is where $x_{1}=x_{2}$ which, using Eq. (3), can be written as

$$
\frac{1}{f}=-\frac{1}{L_{d}}(1+\delta),
$$

with the error for determining the focal length given by

$$
\delta=\frac{\delta x_{0}+\delta x_{0}^{\prime} L_{d}}{x_{0}}
$$

This implies that the greater the initial offset, the smaller the contribution of other error sources to the final focal-length measurement. In other words, to calibrate the rf amplitude with $1 \%$ accuracy it is necessary to have $\delta \leq 1 \%$. In reality, the crossing point is found after a linear fit to the BPM data and thus Eq. (5) gives an upper boundary to the initial offset needed. Equation (5) is also a simplification of the whole system, not taking into account effects like BPM sensitivity to bunch length or energy. However, as discussed in the following sections, only the BPMs closest to the cavities are used, so this problem is not of immediate concern. Deliberately increasing the initial offset should be avoided: this may introduce additional problems such as nonlinear terms and coupling effects, which are not considered in the linear theory developed thus far. As trajectory correction and phase scans are performed with low-current beam pulses, the losses are not a concern from a machine protection point of view. However, with significant losses, the beam center-ofmass measurement becomes unreliable, which has a negative impact on the accuracy of the method.

\section{A. Cavity errors}

The scanning method at this point is fairly compact and simple, but once cavity errors (tilts and offsets) are included, some care must be taken. For the case of a cavity alignment error $\left(-\Delta_{\mathrm{rf}}\right)$ it is clear that each trajectory will experience a different focusing force. The effect of a cavity tilt $(-\theta)$ however, is not so simple, but can be modeled for $\theta \ll 1$ by adding an extra term to the cavity matrix.

To include these misalignments, we add the cavity errors (with opposite sign) to the initial beam centroid coordinates, propagate through the cavity, and remove these same errors afterwards. Using a matrix for the cavity which assumes a drift-kick-drift lumped effect at the cavity center, and keeping only elements to first order on the cavity amplitude and phase, the complete equation is

$$
\begin{aligned}
{\left[\begin{array}{l}
x \\
x^{\prime}
\end{array}\right]=} & M_{\mathrm{cav}}\left[\begin{array}{c}
x_{0}+\Delta_{\mathrm{rf}}-\theta L_{\mathrm{cav}} / 2 \\
x_{0}^{\prime}+\theta
\end{array}\right] \\
& -\left[\begin{array}{cc}
1 & L_{\mathrm{cav}} \\
0 & 1
\end{array}\right]\left[\begin{array}{c}
\Delta_{\mathrm{rf}}-\theta L_{\mathrm{cav}} / 2 \\
\theta
\end{array}\right],
\end{aligned}
$$




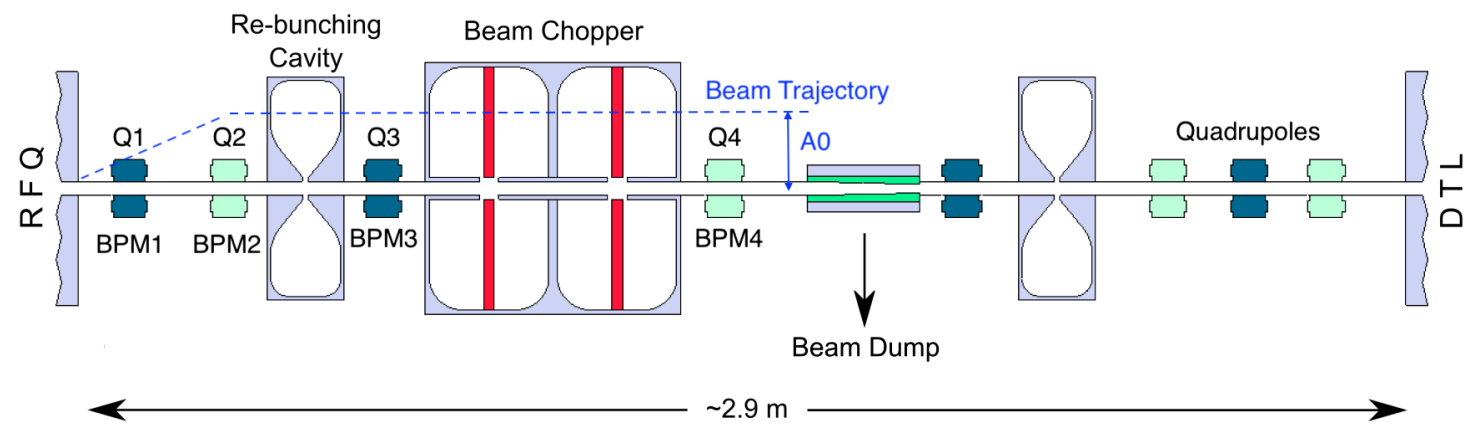

FIG. 2. Layout of J-PARC's MEBT1 section, showing the positions of the buncher cavities and quadrupoles. The BPMs and steerers are colocated with the quadrupoles.

where

$$
\begin{aligned}
M_{\mathrm{cav}}= & {\left[\begin{array}{cc}
1 & L_{\mathrm{cav}} / 2 \\
0 & 1
\end{array}\right]\left[\begin{array}{cc}
1-K_{+}(V) \cos \phi & 0 \\
F(V) \sin \phi & 1-K_{-}(V) \cos \phi
\end{array}\right] } \\
& \times\left[\begin{array}{cc}
1 & L_{\mathrm{cav}} / 2 \\
0 & 1
\end{array}\right],
\end{aligned}
$$

where $L_{\text {cav }}$ is the cavity length. The coefficients $K_{ \pm}$are described in the Appendix.

In order to simplify the expression above, it is assumed here that one may control the trajectory at the entrance of the cavity such that the initial trajectory angles can be corrected to zero $\left(x_{0}^{\prime}=0\right)$ and that there is no coupling between the transverse planes. In order to create the required bump, it is possible to use two steerers before the cavity along with a pair of BPMs, one before and another after the scanned cavity. If an automatic trajectory correction algorithm is already in place, the bump can be created by adding the same offset to the reference position of the same pair of BPMs and allow the algorithm to correct the trajectory.

Considering a drift with length $L_{\mathrm{bpm}}$ leading to a BPM, the measured trajectory is

$$
\begin{aligned}
x_{\mathrm{BPM}}= & x_{0}-\left(x_{0}+\Delta_{\mathrm{rf}}\right) K_{+}(V) \cos \phi \\
& +\left(L_{\mathrm{bpm}}+\frac{L_{\mathrm{cav}}}{2}\right)\left[\left(x_{0}+\Delta_{\mathrm{rf}}\right) F(V) \sin \phi-\theta K_{-}(V) \cos \phi\right] .
\end{aligned}
$$

A second step to further simplify the expression above is to perform the measurement for two initial offsets, $x_{10}=$ $-x_{20}=x_{0}$, and look at the difference and sum trajectories

$$
\begin{aligned}
x_{\text {diff }} & =\frac{x_{1}-x_{2}}{2} \\
& =x_{0}+x_{0} L_{d} F(V) \sin \phi-x_{0} K_{+}(V) \cos \phi, \\
x_{\text {sum }} & =\frac{x_{1}+x_{2}}{2} \\
& =\Delta_{\mathrm{rf}} L_{d} F(V) \sin \phi-\left[\theta L_{d} K_{-}(V)+\Delta_{\mathrm{rf}} K_{+}(V)\right] \cos \phi,
\end{aligned}
$$

where $L_{d}=L_{\mathrm{bpm}}+L_{\mathrm{cav}} / 2$ is the distance from the cavity center to the BPM center and the contributions of cavity offset and tilt are clearly separated. It is possible to calibrate the rf amplitude and phase using Eq. (8) and to estimate the cavity errors for each plane using Eq. (9). The same procedure can be carried out for the vertical plane.

At this point a digression is important, the cavity misalignment errors measured by this method are exact only if the default trajectory in the linac is a straight line passing through the center of all elements. In reality this is not the case and the trajectory can be defined in different ways. One of the most common methods is to define the center of the BPM with respect to the center of the nearby quadrupoles, which renders the default trajectory to be composed of piecewise lines connecting the centers of adjacent BPMs. In this latter case, using one BPM before and one after the cavity, it is possible to measure the misalignment of the cavity with respect to this particular trajectory. For the cases where alignment and magnetic center errors are within hundreds of micrometers but misalignment errors of the super conducting (SC) cavities are expected to be one order of magnitude higher or more, knowing the relative misalignments and feeding them back into the models can considerably improve the quality of the predictions.

\section{PROOF OF PRINCIPLE}

J-PARC is a joint laboratory between the Japan Atomic Energy Agency (JAEA) and the High Energy Accelerator Research Organization (KEK). The facility is situated at the JAEA/Tokai site. An $H^{-}$beam with a peak current of $50 \mathrm{~mA}$ and a pulse length of up to $500 \mu \mathrm{s}$ is accelerated up to $400 \mathrm{MeV}$ with a repetition rate of $25 \mathrm{~Hz}$. The linac consists of a volume-production type $H^{-}$ion source, a $3 \mathrm{MeV}$ RFQ, a $50 \mathrm{MeV}$ DTL, a $191 \mathrm{MeV}$ separated-type DTL and a $400 \mathrm{MeV}$ annular coupled structure linac. Between the RFQ and DTL sections lies the MEBT1 which is a compact beam transport line, as shown in Fig. 2. Its main functions are to chop, characterize, and match the beam for subsequent acceleration in the DTL. Eight quadrupole magnets and two buncher cavities provide 
TABLE I. Parameters for the first MEBT buncher cavity.

\begin{tabular}{lcc}
\hline \hline Parameter & Value & Unit \\
\hline Input energy & 3.0 & $\mathrm{MeV}$ \\
rf frequency & 324 & $\mathrm{MHz}$ \\
Design accelerating voltage $\left(E_{0} T L\right)$ & 164 & $\mathrm{kV}$ \\
Transit time factor & 0.55 & $\ldots$ \\
Gap length & 18 & $\mathrm{~mm}$ \\
\hline \hline
\end{tabular}

the transverse and the longitudinal matching. The MEBT1 is equipped with a suite of beam diagnostics including beam current monitors, beam position monitors and wire scanners.

A series of measurements using the position-based cavity tuning was performed at J-PARC in March 2019 and compared with conventional ToF and signature matching measurements $[2,3,7,9]$ for the first buncher cavity in the MEBT1 section. Those measurements corresponds to a first proof-of-principle for the scanning method and are presented in this section. For all measurements, a beam pulse of $50 \mu$ s with a current of $50 \mathrm{~mA}$ was used.

\section{A. Measurement setup}

For this test, the second and third quadrupoles $\left(Q_{3}\right.$ and $Q_{4}$ in Fig. 2) in the MEBT1 section current were set to zero, turning the lattice downstream into a simple drift-cavitydrift system. Just after the $B P M_{4}$ there is a beam dump, consisting of a pair of scrapers, which were closed during the measurement so that no beam would reach the DTL entrance. The main parameters for the buncher cavities, relevant for the simulations, are listed in Table I.

In order to create the necessary trajectory bumps for the amplitude and phase measurements, a reference a trajectory obtained after a beam based alignment of the quadrupoles was used. Over this trajectory, the steerers, integrated in the first two quadrupoles, were used to manually create trajectory bumps with a given offset and zero angle going through the buncher cavity.

At J-PARC, in order to avoid transporting unaccelerated particles through the MEBT1 and into the DTLs, the whole RFQ and the first two quadrupoles in the MEBT1 section are intentionally misaligned by $-1 \mathrm{~mm}$ in the horizontal plane. This offset made the measurement on the horizontal plane especially difficult. In addition, the chopper creates a substantial aperture restriction in the horizontal plane, with a horizontal aperture of $13 \mathrm{~mm}$ (in contrast with the $20 \mathrm{~mm}$ aperture for the vertical). In light of these issues, and owing to time constraints, scans were performed only on the vertical plane.

\section{B. Phase scan}

The first step here was to find the phase for maximum transverse focusing, i.e., 90 degrees from the linac's definition of zero phase, which in the longitudinal plane
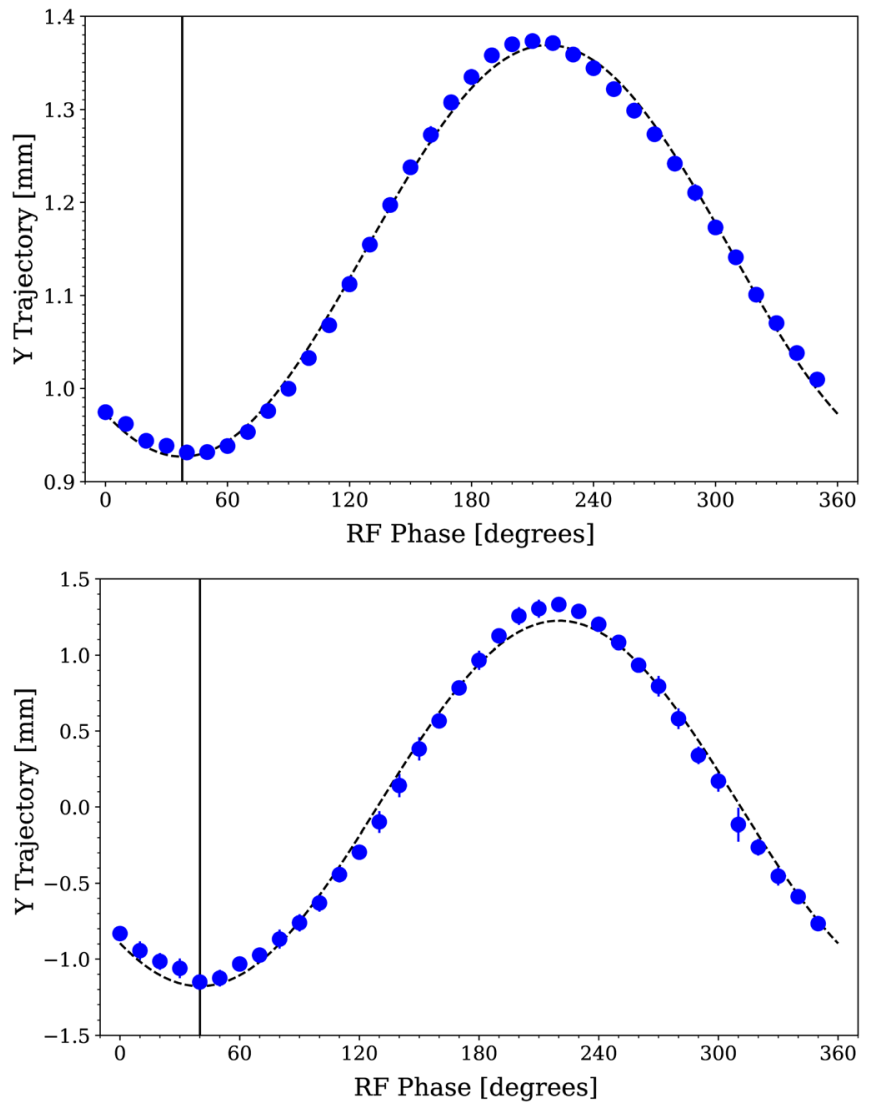

FIG. 3. Trajectory measured at $B P M_{3}$ (top) and $B P M_{4}$ (bottom) in the MEBT1 section. The dots are data points, the dashed lines are the sinusoidal fit, and the vertical line represents the debunching phase. The error bars are standard deviations from 10 measurements.

corresponds to the defocusing, or debunching phase. At this stage, any bump can be used without much care of canceling angles or knowing the initial conditions precisely.

Figure 3 shows this type of simple scan and the corresponding sinusoidal fit to the BPM data. The measured phase was $39.9 \pm 0.5$ degrees using $B P M_{3}$ and $40.1 \pm 0.8$ degrees using $\mathrm{BPM}_{4}$, while the phase measured from the traditional phase scan, using ToF, was $39.9 \pm 1.0$ degrees.

It is worth noting that the reading from $B P M_{4}$ is not as reliable as $B P M_{3}$ : Since the two quadrupoles downstream from the buncher were turned off, the beam loss at the chopper was on average $38 \%$, making the measurement at $B M_{4}$ less reliable; this can explain the larger difference in phase observed. From this point forward, only a comparison of $B P M_{3}$ measurements with ToF results will be presented (although some results from $B P M_{4}$ will still be shown in order to illustrate the rf focusing effect further away from the cavity).

\section{Amplitude scan}

Four bumps with different amplitudes were defined for the amplitude scans, as shown by a dashed blue line in 
TABLE II. Vertical trajectory-bump amplitudes in the MEBT1.

\begin{tabular}{lc}
\hline \hline Name & Amplitude $A_{0}(\mathrm{~mm})$ \\
\hline Bump 1 & $0.57 \pm 0.01$ \\
Bump 2 & $1.14 \pm 0.02$ \\
Bump 3 & $-0.59 \pm 0.01$ \\
Bump 4 & $-1.13 \pm 0.01$ \\
\hline \hline
\end{tabular}

Fig. 2. For all bump amplitudes, the steerers preceding the buncher cavity were set such that the angle of the beam traversing the cavity was as close as possible to zero, this was ensured by making the readings in the following BPMs equal. The amplitude of each bump can be found in Table II which were measured with the cavity and the downstream quadrupoles turned off. For the amplitude scans, the cavity phase was set to 90 degrees, which yields the maximum transverse-focusing kick.

The amplitude of the cavity was scanned for each bump, the measured trajectory was fitted with a straight line, and the crossing points for all four bumps were calculated. The results are shown in Fig. 4. From the model, the crossing amplitude for each BPM can be calculated, and this is the amplitude that makes the focal length of the cavity equal to
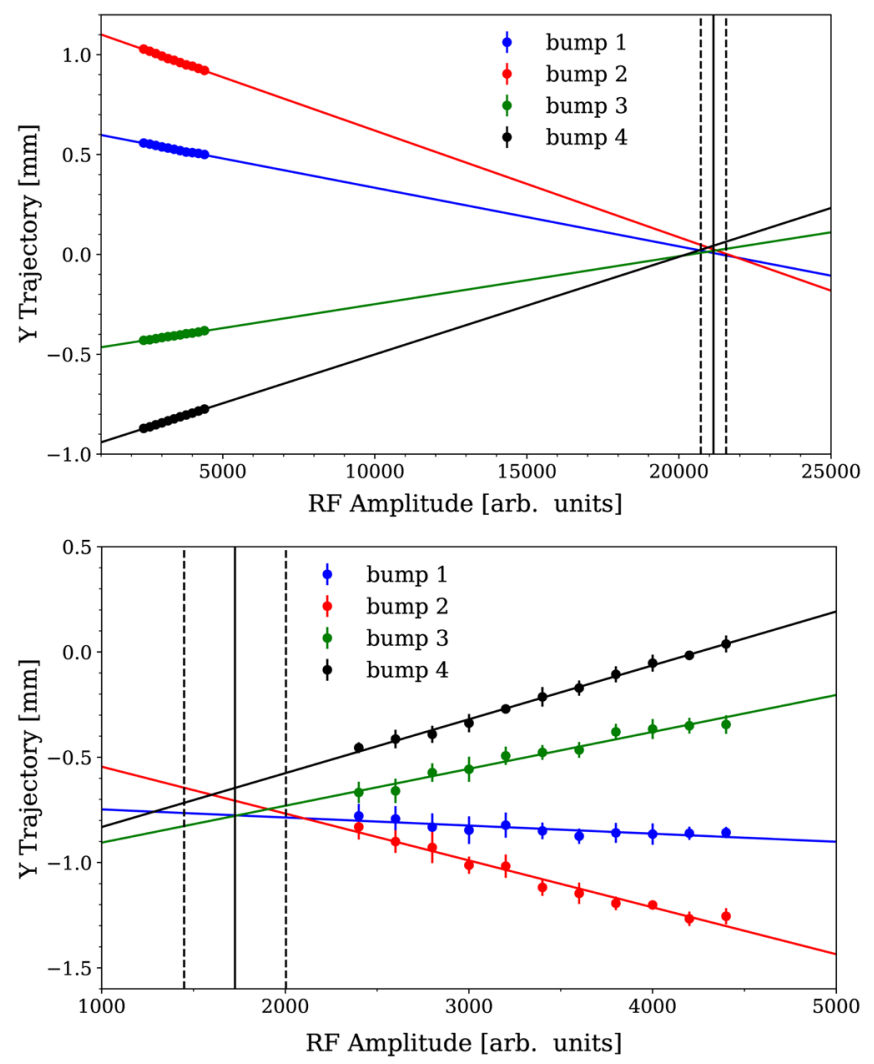

FIG. 4. Vertical trajectory during the rf amplitude scan for four different initial bump configurations for $\mathrm{BPM}_{3}$ (top) and $B P M_{4}$ (bottom). The dots are the measurements and the lines are linear fits to the data. The solid vertical black lines represent average crossing value; dashed lines indicate $\pm 1 \sigma$.
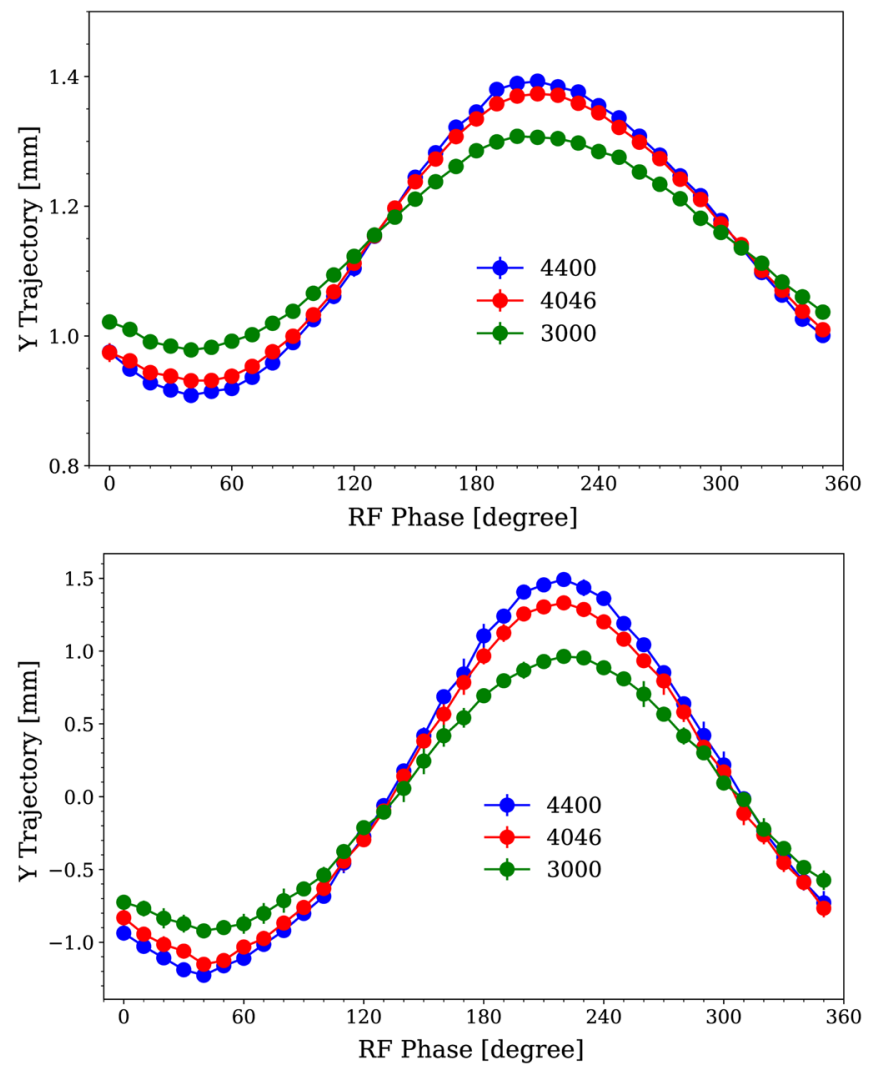

FIG. 5. An example of a phase scan for three different cavity amplitudes measured at $\mathrm{BPM}_{3}$ (top) and $\mathrm{BPM}_{4}$ (bottom). The amplitude values are those set in the cavity controller and have no direct physical meaning. The default input amplitude for this cavity is 4046 arb. units.

its distance to the BPM in question. This amplitude for having the focal point at $B P M_{3}$ is $E_{0} T L=1198 \mathrm{kV}$ and for $B P M_{4}$ it is $E_{0} T L=217 \mathrm{kV}$. From the measurement the crossing amplitude $\left(A_{\text {cross }}\right)$ is $21134 \pm 400$ and $1725 \pm 300$ arb. units for $B P M_{3}$ and $B P M_{4}$ respectively. The calibration factor between the cavity input amplitude and the effective accelerating voltage is then the ratio between those two quantities, $\alpha=E_{0} T L / A_{\text {cross }}$, for each BPM.

Another way to calibrate the cavity amplitude is to perform phase scans at different cavity amplitudes and check the maximum trajectory displacement. From Eq. (3) this corresponds to the term proportional to $1 / f$, and the maximum trajectory variation is given by $F(V) L_{d} A_{0}$, where $A_{0}$ is the bump amplitude as shown in Fig. 2. The amplitude calibration between the trajectory and $E_{0} T L$ can be found from simulation, which is a similar approach as the signature matching used in ToF based calibration. A set of three phase scans was performed for different cavity amplitudes using bump 2. The results for each phase scan are shown in Fig. 5 and the $B P M_{3}$ fit is shown in Fig. 6 .

A summary of the results for all amplitude measurements and the corresponding calibration constants are in Table III. As expected, the trajectory-crossing measurement is less 


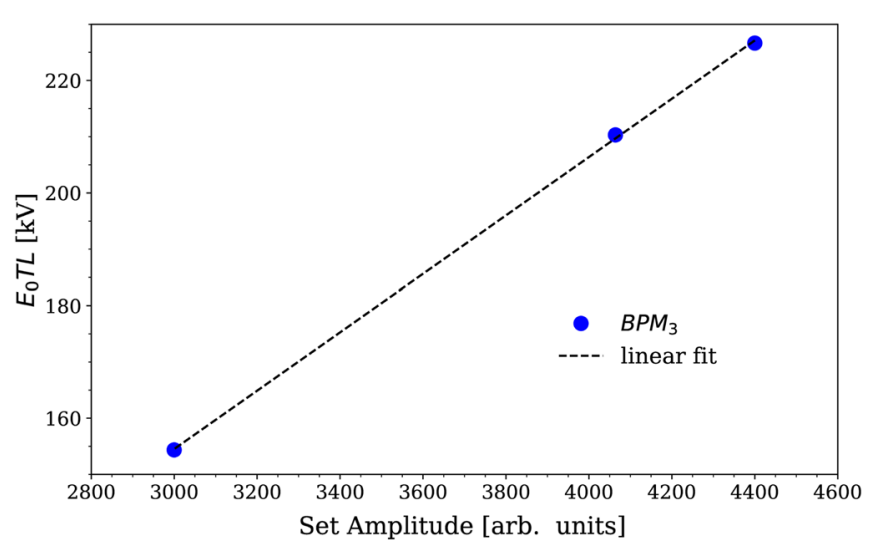

FIG. 6. Calibration relation between cavity input amplitude and $E_{0} T L$. The dashed line is a linear fit to the data.

accurate than the signature matching. Although the calibration from the position-based measurements relies on just three amplitude points (while the ToF calibration uses ten or more) the results are still similar, which is promising for the position-based method. In order to have a more accurate comparison, it would be necessary to perform position and ToF measurements at the same time and with the machine at the same conditions, which was not possible during this first test.

\section{Cavity misalignment}

Once phase and amplitude are calibrated, it is also possible to extract the cavity misalignment. For the JPARC data, since the measurements were performed on the vertical plane, the vertical offset and the pitch angle can be estimated. All the phase scans were performed at the same cavity amplitude $E_{0} T L=164 \mathrm{kV}$, which is the default value for operation.

The first step in this process is to calculate the sum and difference trajectory for each pair of bumps, as shown in Fig. 7. In this figure, the error bars from the bump amplitude are the standard deviation from 10 consecutive measurements and reflect only the BPM precision. Since in this case the initial trajectory angles were close to zero after $Q_{2}$ - and the bump amplitudes are the same, but with opposite signs-it is possible to use Eqs. (8) and (9). Pairing bumps 1 and 3 and bumps 2 and 4, the sum and difference trajectories are shown in Fig. 8. The results from the fit of Eq. (8) and (9) are then summarized in Table IV. The error bars are based on the fit, and each data point

TABLE III. MEBT1 buncher-amplitude calibration results. The calibration relates $E_{0} T L(V)=\alpha A_{\text {input }}$.

\begin{tabular}{lc}
\hline \hline Measurement type & $\alpha(V)$ \\
\hline Crossing $B P M_{3}$ & $57 \pm 7$ \\
Conventional signature match $\left(B P M_{3}\right.$ only) & $51.8 \pm 0.1$ \\
Standard Signature match using ToF & 44.3 \\
\hline \hline
\end{tabular}

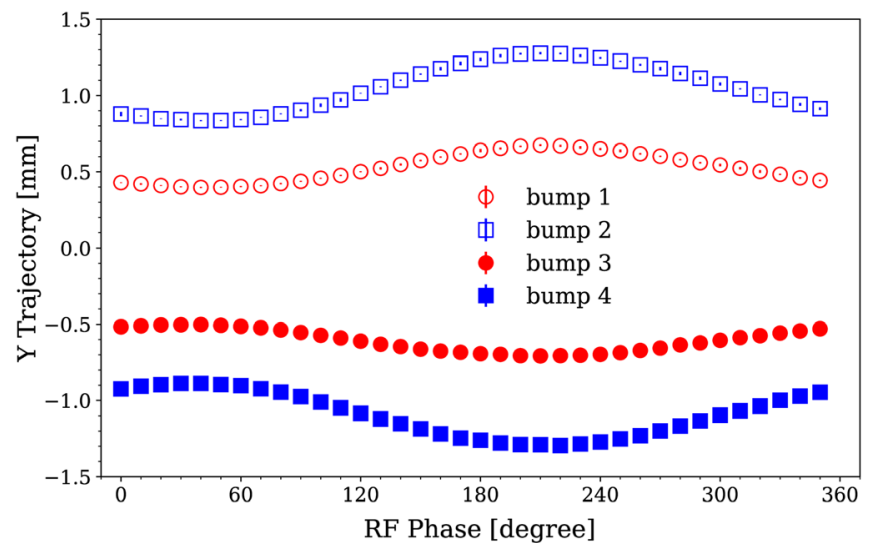

FIG. 7. Phase scan for different bump amplitudes measured for $B P M_{3}$. The cavity was set to its default amplitude of 4046 arb. units, which corresponds to $E_{0} T L=164 \mathrm{kV}$.

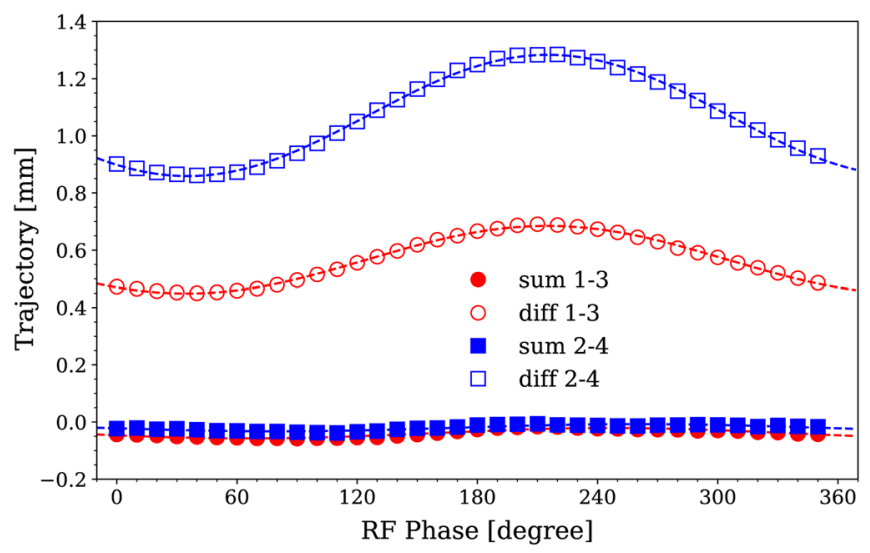

FIG. 8. Sum and difference trajectories derived from the measurements in Fig. 7 and the respective fit using Eq. (8) and (9).

shown is an average of ten measurements. Note that the reconstructed bump values agree well with the values measured at the BPMs, and from the misalignment results it is possible to see that the cavity is well aligned with the beam trajectory.

\section{SINGLE CELL VERSUS MULTICELL CAVITIES}

The European Spallation Source (ESS), currently under construction in Lund, Sweden, will be a spallation neutron source driven by a superconducting proton linac [10]. The linac in its final configuration will accelerate a proton beam

TABLE IV. Cavity misalignment results.

\begin{tabular}{lccc}
\hline \hline Measurement set & $\Delta_{\mathrm{rf}}(\mathrm{mm})$ & $\theta(\mathrm{mrad})$ & $x_{0}(\mathrm{~mm})$ \\
\hline Bump 1 and 3 & $0.08 \pm 0.01$ & $3.4 \pm 0.3$ & $0.6 \pm 0.1$ \\
Bump 2 and 4 & $0.04 \pm 0.01$ & $3.2 \pm 0.3$ & $1.1 \pm 0.1$ \\
\hline \hline
\end{tabular}




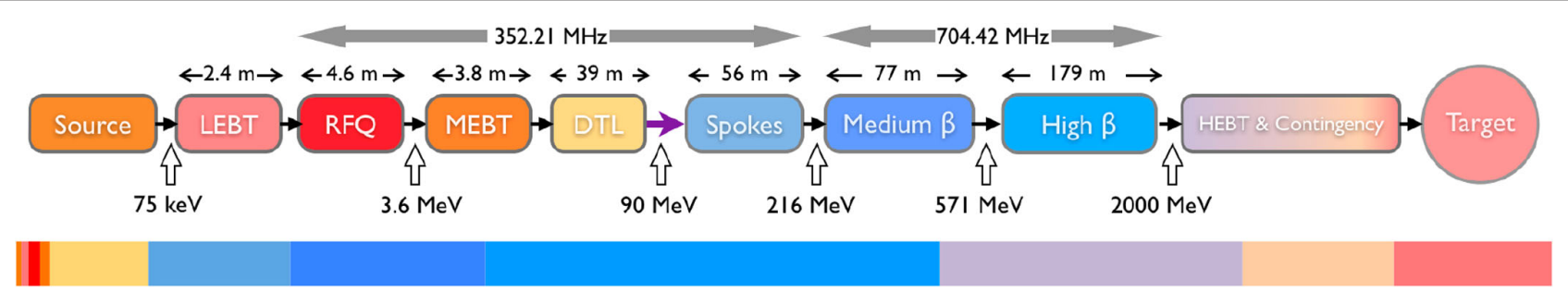

FIG. 9. Block layout of the ESS accelerator from source to target (top) and its scaled version (bottom).

with a $62.5 \mathrm{~mA}$ peak current and $4 \%$ duty cycle $(2.86 \mathrm{~ms}$ pulse length at $14 \mathrm{~Hz}$ ) up to $2 \mathrm{GeV}$ and produce an unprecedented $5 \mathrm{MW}$ average beam power. The superconducting linac has a normal conducting linac (NCL) as its injector, which consists of an ion source, radio frequency quadrupole (RFQ), drift tube linac (DTL), as well as low and medium energy beam transports (LEBT and MEBT). The NCL accelerates the proton beam from $75 \mathrm{keV}$ to $90 \mathrm{MeV}$. The superconducting linac (SCL) is composed of 26 double-spoke cavities; 36 medium- $\beta$ elliptical cavities, and 84 high- $\beta$ elliptical cavities.

A block layout of the ESS accelerator is shown in Fig. 9. The requirements on phase and amplitude accuracy at ESS are $1^{\circ}$ and $1 \%$, respectively [10]. Like many modern hadron linacs, a large part of the ESS linac consists of superconducting, multicell cavities. In principle, the same concept for the position-based phase scan used for a single gap can be used for these multicell cavities. However, a few details have to be considered before applying Eq. (3). As stated earlier, modeling the lattice with elements that represent the nonlinear fields of the components is one way to avoid large errors without resorting to complicated analytical analysis.

In this section, a set of simulations for a multicell type cavity is shown. For the trial simulation, the parameters for the first medium beta (MB) cavity at ESS were used. This cavity is a six-cell super-conducting rf cavity with a maximum gradient of $9 \mathrm{MV} / \mathrm{m}$, working at $704.42 \mathrm{MHz}$. The input beam energy used was $216 \mathrm{MeV}$ and only the zerocurrent case was considered; the errors used were those listed in Table V. The tracking code used was TraceWin [11] with 200,000 macro-particles per bunch and on-axis field maps for the rf fields; TraceWin uses Bessel functions to estimate the off-axis field values. The cavity misalignment calculation is not presented for this case and it is assumed it

TABLE V. Errors and misalignments used for the simulations of the multicell cavity.

\begin{tabular}{lcc}
\hline \hline Element & Parameter & Error \\
\hline Quadrupole & Magnetic center & $0.2 \mathrm{~mm}$ \\
& Roll angle & $0.6 \mathrm{deg}$ \\
& Gradient & $0.5 \% \mathrm{deg}$ \\
BPM [12] & Position accuracy & $100 \mu \mathrm{m}$ \\
& Angle accuracy & $100 \mu \mathrm{rad}$ \\
\hline \hline
\end{tabular}

was performed beforehand, thus only residual misalignments between the cavity, BPMs, and the quadrupole are considered.

\section{A. Dependencies of the method}

Equation (2) is a valid approximation for a single-gap cavity but it cannot be used directly to describe the multicell cavities accurately. For the multicell case, an expression similar to Eq. (2) can be used if some additional factors are included. Multicell cavities with constant cell length are designed for a specific particle velocity $\beta_{g}$. In order to accelerate the beam, each cell has a length of $\beta_{g} \lambda / 2$ (in $\pi$ mode structures), so that a bunch traversing the cavity always sees the accelerating phase of the electric field. However, when $\beta \neq \beta_{g}$ the phase of the beam relative to the electric field slips as it travels along the cells and the bunch is only exactly "on phase" in the geometric center of the structure [13]. For this reason, the designation "synchronous phase" $\phi_{s}$ no longer applies and is usually replaced by the mean phase of the reference particle $\phi_{r}$. This effect of phase slippage has consequences on the maximum energy gain a cavity can provide, and also on the transverse focusing effect.

The reduction in energy gain for $\beta \neq \beta_{g}$ due to phase slippage is given by evaluating the transit-time factor integral for the appropriate velocity. For a simplified case, assuming constant electric fields on axis, and neglecting the effects of acceleration

$$
\begin{aligned}
\Delta W & =q \int_{-L / 2}^{L / 2} E(r, z, t) d z \approx q E_{0} T(\beta) \\
& =q \int_{-L / 2}^{L / 2} E(t=0, z)\left(\frac{\omega z}{\beta c}\right) \cos \left(\frac{\omega z}{\beta c}+\frac{\kappa(n) \pi}{2}\right) d z,
\end{aligned}
$$

with

$$
\kappa(n)= \begin{cases}0 & \mathrm{n} \text { is odd } \\ 1 & \mathrm{n} \text { is even }\end{cases}
$$

where $\mathrm{n}$ is the number of cells in the structure. For this simplified case it is possible to rewrite the energy gain as:

$$
\Delta W=q E_{0} T(\beta) f(n) \cos \left(\phi-\phi_{r}+\frac{\kappa(n) \pi}{2}\right),
$$


where the transit-time factor depends on the beam energy and the form factor $f$ dictates the dependency on the number of cells $(n)$ in the cavity. This form factor does not have a simple form, but can be also obtained numerically as the reference particle mean phase $\phi_{r}$. The same reasoning can be applied to the transverse focusing effect of the cavity, and each cell can be treated as a single gap with different transit times and phases. However the transverse focusing effect on the trajectory is not just a simple addition of the effect on each cell. For the simplest case of a two-cell structure, for example, we can derive the beam parameters at the end of the cavity-drift system, assuming a lump effect at the center of each gap:

$$
\begin{aligned}
x= & x_{0}+x_{0}\left(L_{b p m}+\frac{L_{\text {cell }}}{2}\right)\left[L_{\text {cell }} F_{1} F_{2}+F_{1}+F_{2}\right] \\
& +x_{0} L_{\text {cell }} F_{1},
\end{aligned}
$$

where $F_{n}=A_{n} \sin \left(\phi \pm \phi_{n}\right), L_{\text {cell }}$ is the cell length, $L_{b p m}$ is the drift from the center of the cavity to the measurement position and we further assumed the initial particle angle was zero $\left(x_{0}^{\prime}=0\right)$. Similar to the procedure for the energy, it is possible to rewrite this expression in a way it resembles the single gap case. However this is not just a simple addition and cross terms arise. The final expression is, using the same format as Eq. (2):

$$
x=x_{0}+x_{0} L_{b p m} F(V) g(n) \sin \left[\phi-\phi_{r}+\theta(n)\right] .
$$

and

$$
F(V)=-\frac{\pi q V T(\beta)}{m c^{2} \lambda(\gamma \beta)^{3}}
$$

Note that the average transit time $T(\beta)$ is used, depending only on the initial energy. Then in addition to an amplitude form factor $g(n)$, a phase offset $\theta(n)$ is needed. The phase offset has a very weak dependency on cavity amplitude, but depends strongly on the cavity cell number $(n)$. Unlike the single-gap case, for multicell cavities the energy curve and trajectory-signature curves are not exactly 90 degrees apart; this offset has to be obtained from numerical simulations. For our test case, the total offset is calculated to be $1.92 \pm$ 0.02 degrees across the whole amplitude range.

The position-based cavity tuning method relies on the transverse focusing effect of the rf cavity, which is energy dependent. The focal length for the cavity is given by Eq. (2). As the beam energy/momentum increases this (de) focusing effect becomes weaker; if a quadrupole is located between the cavity and the BPM during a phase scan, the focusing force of the quadrupole (also a function of energy) will cause the whole trajectory curve to shift by another constant phase with respect to the energy curve. For the first MB cavity at ESS, the quadrupole located between the

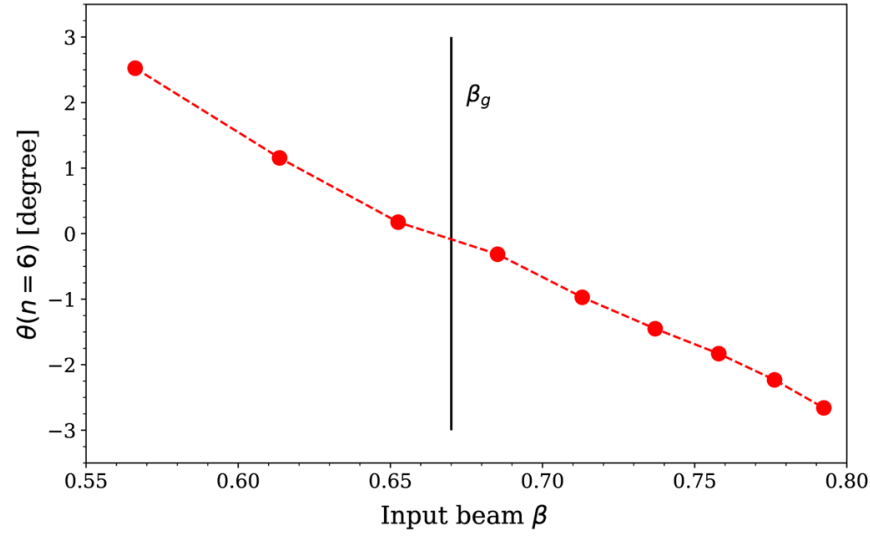

FIG. 10. Example of the phase offset between trajectory and energy as a function of input beam energy for a six-cell cavity with a design geometrical $\beta_{g}$ of 0.67 (vertical full line). The operational range for this specific cavity is between a $\beta$ of 0.58 to 0.78 and for that the phase offset is within \pm 2 degrees.

cavity and the first BPM adds an extra phase of 0.35 degrees.

Another effect to consider is a possible energy error of the incoming beam. For this first MB cavity, the phase offset between the trajectory and energy varies by 0.05 degree per $\mathrm{MeV}$ of beam-energy offset and is well below the requirements on the phase accuracy (if we assume a possible input energy variation of $10 \mathrm{MeV}$ ). For the amplitude, the error scales as $0.5 \%$ error per $\mathrm{MeV}$ of energy offset and for large energy uncertainties the total amplitude error will exceed the $1 \%$ limit.

Continuing with the first ESS MB cavity, the phase offset dependence for a larger range of input beam energies, corresponding to its operational energy, was also calculated and is shown in Fig. 10. The variation of the offset is smooth; and near the energy corresponding to the geometrical beta the offset is zero, as expected.

\section{B. Sensitivity to lattice imperfections}

Two possible settings for the position-based phase scan were analyzed: (1) horizontal displacements with no focusing element between the cavity and BPM (2) horizontal displacements with a single quadrupole between the cavity and the BPM.

For each setting, a simulated measurement of a phase scan was simulated for a single cavity amplitude, corresponding to $85 \%$ of the maximum amplitude, for two different initial offsets of +1 and $-1 \mathrm{~mm}$; in both cases the initial angle is set to zero. The results are the standard deviation of 50 cases using the errors described in Table V.

An example of a simulated phase scan is shown in Fig. 11. From the sinusoidal fit it is possible to determine the relative phase between cavity rf and beam, and then to the extract the trajectory's relative amplitude and offset. For comparison, in the same figure, the beam centroid energy 


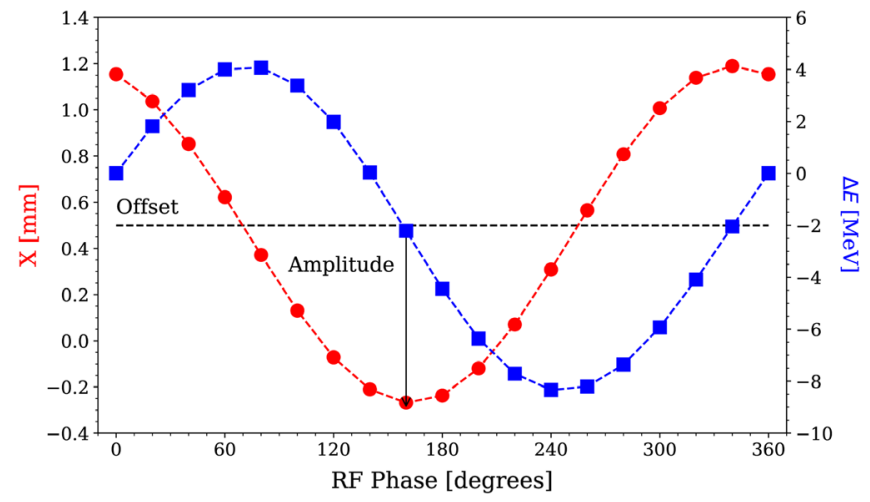

FIG. 11. Example of a phase scan for a six-cell cavity with a quadrupole in between cavity and BPM. The left axis shows the measured trajectory and the right axis shows the beam centroid energy variation. In addition to the relative phase, the trajectory amplitude and offset can give valuable information about the cavity settings.

deviation is also shown and can be seen that the two curves are almost 90 degrees out of phase.

For the phase, the statistical sensitivity study showed that it is possible to determine the relative phase between cavity and beam within the required one-degree limit for all cases, see Table VI. The presence of a quadrupole between the scanned cavity and the BPM has a larger effect on the accuracy of the phase than the amplitude, which was expected since the amplitude is a relative measurement and the quadrupole is a static effect that is removed once the relative amplitudes are fitted. Although the quadrupole causes an additional error in the phase offset, both results are below the required one-degree limit.

In order to perform a full amplitude calibration, the relation between the effective amplitude seen by the beam and the maximum amplitude deviation on the beam trajectory was obtained from a simulation without errors (Fig 12). For machines with errors, as described in Table V, it was possible to determine the rf amplitude to within 5\% with two sets of scans at one cavity amplitude but two different initial offsets as shown in Table VI.

It is possible to reduce the uncertainty in amplitude further by examining the trajectory offset measured at the BPM. The offset measured for case 1, for example, should be the same as the initial offset. If it is not the same, since the position is a direct measurement of the BPM, it is possible to attribute the deviation to an initial residual angle. Correcting for this initial angle, for case 1, it was

TABLE VI. Medium-beta position-based scan results with errors in the alignment of quadrupoles and BPM readings.

\begin{tabular}{lcc}
\hline \hline Case & Phase error (deg) & Amplitude error (\%) \\
\hline Quadrupole OFF & 0.7 & $5.2(3.8)$ \\
Quadrupole ON & 0.9 & 5.4 \\
\hline \hline
\end{tabular}
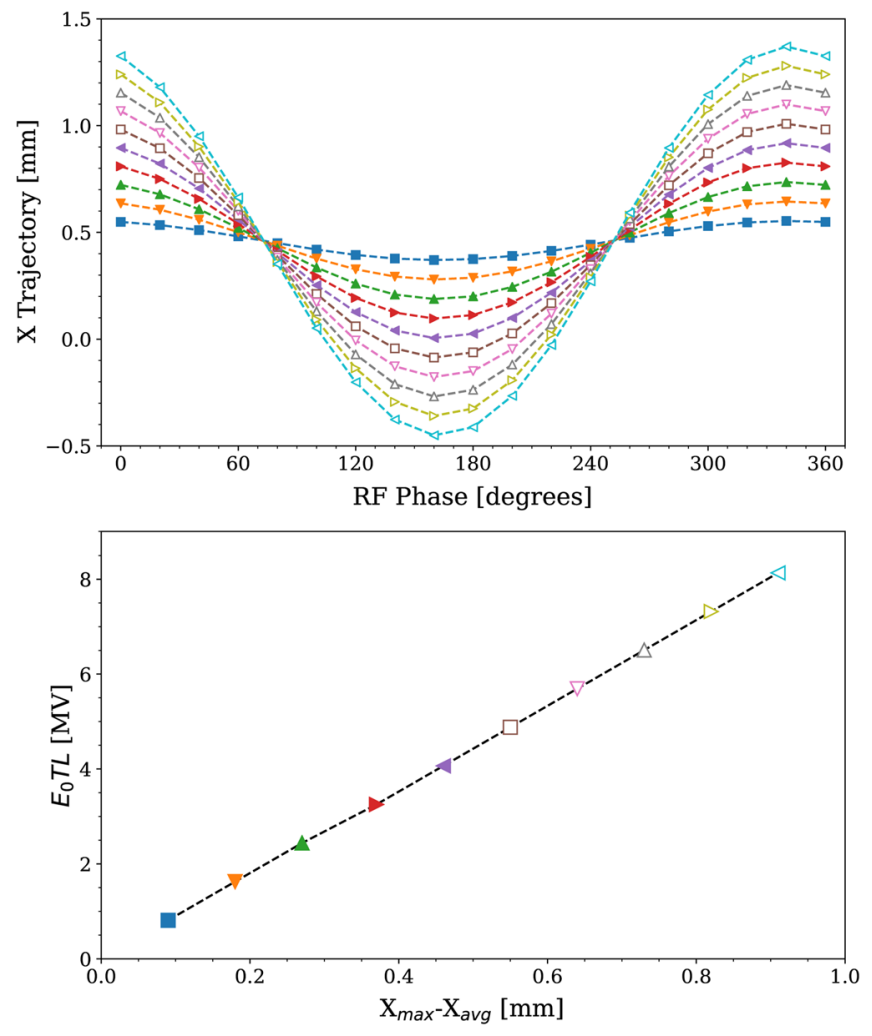

FIG. 12. Calibration using the present model for maximum beam trajectory deviation during a phase scan as a function of the effective acceleration seen by the beam, $E_{0} T L$. The quadrupole between the cavity and the BPM was turned on. Top: trajectories for various cavity amplitudes ranging from $10 \%$ to $100 \%$ of the design amplitude of $9 \mathrm{MV} / \mathrm{m}$. Bottom: dependence of the cavity amplitude on the measured trajectory amplitude, defined as the maximum trajectory displacement minus the average trajectory measured at the BPM.

possible to reduce the rf amplitude error to $3.8 \%$. The dependence of the phase offset between trajectory and energy, and the calibration factor for the amplitude as a function of initial beam centroid angle and beam energy are shown in Fig. 13. The dependence is smooth and a simple polynomial fit is enough to perform the correction.

\section{METHOD LIMITATIONS}

Using position measurements to infer cavity properties has a series of advantages. It was shown that the magnitude of cavity misalignment comes as a byproduct of the measurement itself, without extra costs. Also, there are no further requirements on phase between the equipment (cavity and BPMs) or on the dependencies of the phase measurement with velocity. However some issues exist and are discussed in more detail in the next paragraph.

One of the first limitations of the analytic approach is the reduction of the cavity structure to a simple linear kick. For a good spectrum of cavities, this first order simplification should suffice, as with a buncher. However, more complex structures like DTLs must still be tested with simulation 

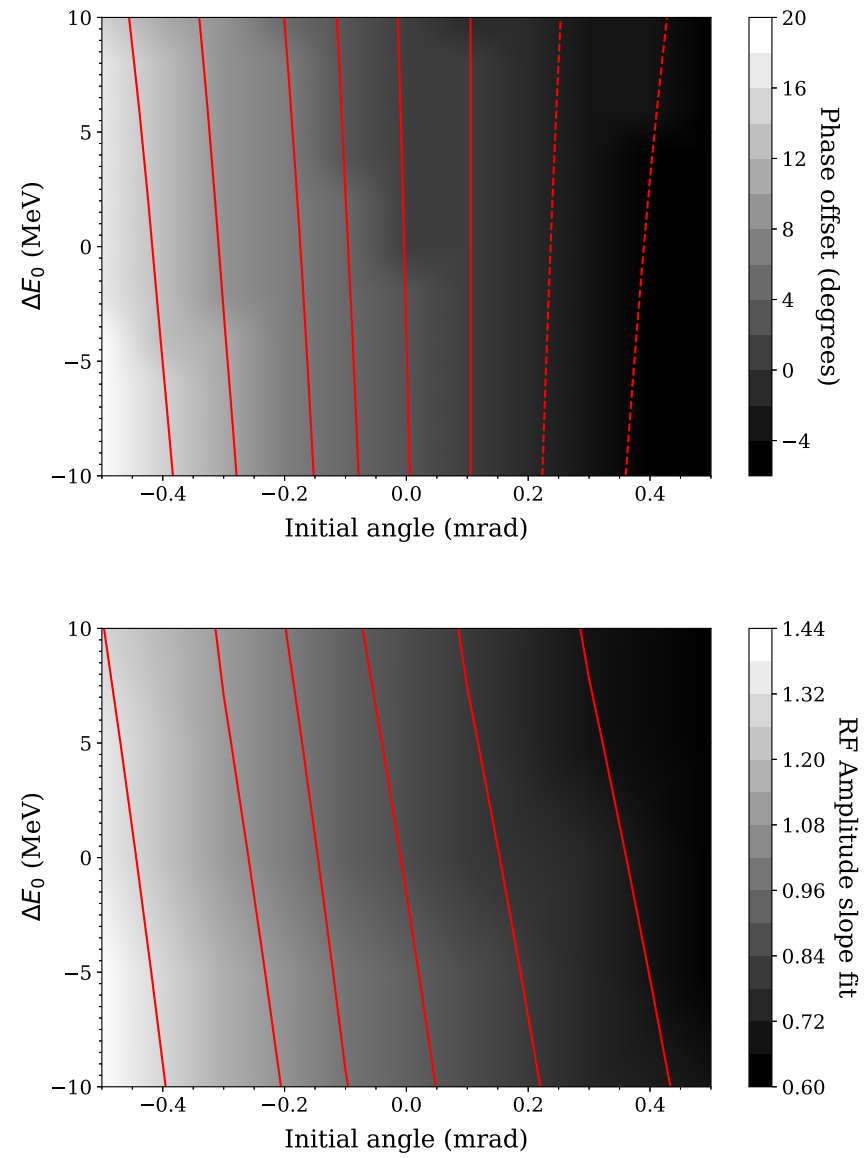

FIG. 13. Dependence of the phase offset and rf amplitude calibration on initial beam energy and initial centroid beam angle. The dependency is well behaved and smooth across a wide range of values. A simple polynomial fit results in residuals on the order of $0.1 \%$. Note that the dependence on the energy is rather weak while the initial residual centroid angle is much more prominent. The red lines show equipotentials on the surface plotted showing path of constant offset (top) or constant rf amplitude slope (bottom). Dashed equipotentials correspond to negative values.

and measurement. Position-based cavity tuning, unlike ToF, is not a direct measurement of the beam-energy variation and thus depends on a good model for the machine, especially the cavities.

Another difficulty of the method lies in the initial setup. It cannot be guaranteed that there will be no focusing element between the cavity and the BPM used to record the position. In this case, a work-around exists in which a single focusing element is allowed, as shown in Section IV. However, for this cases the extra offset created and the error in the quadrupole have to be considered. If more focusing elements exist between the cavity and the measurement point, more simulation and study is needed and tighter requirements on the quadrupoles' field quality and alignment and BPM jitters might be needed in order to achieve the precision required.

Finally, given the dependence of the cavity focusing on energy, from Eq. (2), this method is only fitted to hadron linacs, since for electron machines the effect is greatly reduced because the particles reach relativistic velocities, and thus large values of $\beta \gamma$, already at the initial few meters.

\section{CONCLUSIONS}

A new method for tuning the cavities in hadron linacs is proposed, the method was successfully tested at the MEBT1 buncher cavity of J-PARC. The results from the first test are comparable with those obtained from traditional phase-scan measurements. In addition to cavity tuning, this method also provides a direct way to estimate the cavity tilts and offsets with respect to the beam trajectory.

For the ESS linac, a clear advantage of the new method lies in the fact that the phase readings of the BPMs in the MEBT have a velocity dependence and also that during the first part of the commissioning it is possible to tune the bunchers, even if the phase reference line [10] and the BPM system's phase-measurement accuracy are not fully tested. An additional issue for ESS is that all BPM cables travel from the tunnel to the klystron gallery in the same stubs as the rf waveguides and therefore are subject to temperature variation: having a method that does not depend critically on thermal stability to tune the bunchers to begin with is an advantage.

The main limitation of this method is the fact that a clean path, without other focusing elements between the scanned cavity and the measurement point (usually a BPM) is necessary. This might be the most stringent requirement, specially in machines, like ESS, which require extremely low losses. A work-around is suggested in which one quadrupole sits in between, but at the expense of the requiring a precise and accurate model for the quadrupole.

Further development for long structures such as DTLs are foreseen. Although more tests are still necessary, the position-based cavity tuning method showed promising results, and should become a powerful tool to be used in conjunction with other traditional methods in wellestablished machines as well as a viable option for future linacs.

\section{ACKNOWLEDGMENTS}

The authors would like to thank J-PARC for providing the opportunity to test the presented method and for assisting in performing the measurements documented in this work.

\section{APPENDIX: CAVITY TRANSFER MATRIX}

The cavity lumped effect in its center as described in [14] and can be written as

$$
M_{\text {lumped }}=\left[\begin{array}{cc}
1-K_{+}(V) \cos \phi & 0 \\
F(V) \sin \phi & 1-K_{-}(V) \cos \phi,
\end{array}\right]
$$


where

$$
K_{ \pm}(V)=\frac{q V T}{2 m c^{2} \gamma^{3} \beta^{2}}\left(\gamma^{2} \pm \frac{k T^{\prime}}{T}\right)
$$

and where $T^{\prime}=\partial T / \partial k$ is the derivative of the transit time with respect to the cavity wave number $k=2 \pi / \beta \lambda$. In order to estimate the magnitude of the parameters $K_{ \pm}$we will use the calculated field maps for the cavities and solve the following expression

$$
\frac{k T^{\prime}}{T}=-\frac{k \int_{L / 2}^{L / 2} z E(z) \sin (k z) d z}{\int_{L / 2}^{L / 2} E(z) \cos (k z) d z} .
$$

It is possible to solve this integral when $E(z) \approx E_{0}$

$$
\frac{k T^{\prime}}{T} \approx-1+\frac{k L_{\mathrm{eff}}}{2} \frac{1}{\tan \left(k L_{\mathrm{eff}} / 2\right)} .
$$

Finally, changing $L / 2$ to the effective gap length as $L_{\text {eff }}=\beta \lambda / 2$, and using the relation $1 / \tan (\pi / 2)=0$

$$
K_{ \pm}(V)=\frac{q V T}{2 m c^{2} \gamma^{3} \beta^{2}}\left(\gamma^{2} \mp 1\right)
$$

which for beams at low energy (i.e., with $\gamma^{2} \approx 1$ ) implies that the term $K_{+}$can be neglected. For the J-PARC buncher cavity, the ratio $K_{-} / K_{+}$is 6 and it is safe to simplify the cavity matrix to

$$
M_{\text {lumped }} \approx\left[\begin{array}{cc}
1 & 0 \\
F(V) \sin \phi & 1-K_{-}(V) \cos \phi
\end{array}\right] .
$$

Since the cavity has a length $L_{\text {cav }}$, then the full cavity matrix (using a drift-kick-drift approximation) is

$$
\begin{aligned}
M_{\mathrm{cav}}= & {\left[\begin{array}{cc}
1 & L_{\mathrm{cav}} / 2 \\
0 & 1
\end{array}\right]\left[\begin{array}{cc}
1 & 0 \\
F(V) \sin \phi & 1-K_{-}(V) \cos \phi
\end{array}\right] } \\
& \times\left[\begin{array}{cc}
1 & L_{\mathrm{cav}} / 2 \\
0 & 1
\end{array}\right]
\end{aligned}
$$

[1] A. P. Shishlo, Model and Beam Based Setup Procedures for a High Power Hadron Superconducting Linac, in Proc. 27th Linear Accelerator Conf. (LINAC'14), Geneva, Switzerland, Aug.-Sep. 2014, paper MOIOC03, pp. 41-45.

[2] G. Shen and M. Ikegami, Tuning of RF amplitude and phase for the separate-type drift tube linac in J-PARC, Nucl. Instrum. Methods Phys. Res., Sect. A 598, 361 (2009).

[3] G. Shen and M. Ikegami, Tuning of RF amplitude and phase for the drift tube linac in J-PARC, Chin. Phys C, 33, 577 (2009).

[4] G. B. Shen, H. Sako, and S. Sato, RF amplitude and phase tuning of J-PARC SDTL, in Proceedings of the 22nd Particle Accelerator Conference, PAC-2007, Albuquerque, NM (IEEE, New York, 2007), paper TUPAN062, pp. 1529-1531.

[5] A. Feschenko et al., Development and implementation of T procedure for the SNS Linac, in Proceedings of the 21st Particle Accelerator Conference, Knoxville, TN, 2005 (IEEE, Piscataway, NJ, 2005), paper FPAE049.

[6] S. Henderson, I. E. Campisi, J. Galambos, D.-O. Jeon, and Y. Zhang, Spallation Neutron Source superconducting linac commissioning algorithms, in Proceedings of the 21 st Particle Accelerator Conference, Knoxville, TN, 2005 (IEEE, Piscataway, NJ, 2005), paper FPAE058.

[7] J. Galambos, A. V. Aleksandrov, C. Deibele, and S. Henderson, PASTA An rf phase and amplitude scan and tuning application, in Proceedings of the 21st Particle Accelerator Conference, Knoxville, TN, 2005 (IEEE, Piscataway, NJ, 2005), paper FPAT016, pp. 1529-1531.

[8] M. G. Minty and F. Zimmermann, Measurement and Control of Charged Particle Beams, 1st. ed. (SpringerVerlag, Berlin Heidelberg, 2003).

[9] T. L. Owens, M. B. Popovic, E. S. McCrory, C. W. Schmidt, and L. J. Allen, Phase scan signature matching for linac tuning, Part. Accel. 48, 169 (1994).

[10] R. Garoby et al., The European Spallation Source design, Phys. Scr. 93, 014001 (2018).

[11] D. Uriot and N. Pichoff, Status of TraceWin code, in Proc. 6th Int. Particle Accelerator Conf. (IPAC'15), Richmond, VA, USA, May 2015 (JACoW, Geneva, 2015), pp. 92-94.

[12] R. A. Baron, LWU BPMs signal analysis and requirements, ESS internal report.

[13] F. Gerigk, The effect of phase slippage in multicell cavities on longitudinal beam dynamics, Report No. CERN-NUFACT-NOTE 2001-072, 2001.

[14] T. P. Wangler, RF Linear Accelerators, 2nd ed. (WILEYVCH Verlag GmbH \& Co, New York, 2008). 